Pacific Journal of Mathematics

LOPSIDED SETS AND ORTHANT-INTERSECTION BY CONVEX 


\title{
LOPSIDED SETS AND ORTHANT-INTERSECTION BY CONVEX SETS
}

\author{
JIM LAWRENCE
}

\begin{abstract}
Given a subset $L$ of the $2^{d}$ closed orthants in $d$-dimensional Euclidean space, is there a convex set $K$ which intersects those closed orthants in $L$, while missing those not in $L$ ? A strong combinatorial condition on $L$, which is necessary for the existence of such a convex set, is exhibited. This condition is studied and its close connections with the theory of oriented matroids are examined. The sets $L$ satisfying this condition the "lopsided" sets - have a rich combinatorial structure which can be exploited in the study of convex sets and systems of linear inequalities.
\end{abstract}

1. Introduction. Let $E$ be a finite set and denote by $V(E)$ the vector space of all real-valued functions $f$ on $E$. Let $C(E)$ denote the subset of $V(E)$ consisting of all functions $f$ from $E$ to the set $\{-1,1\}$, so that $C(E)$ is the set of vertices of a cube in $V(E)$. If $f$ is in $C(E)$, then the set:

$$
O(f)=\{g \in V(E): g(e) f(e) \geq 0, \text { for each } e \in E\}
$$

is the closed orthant of $V(E)$ which contains $f$.

If $K$ is a convex subset of $V(E)$, let $L(K)$ denote the set:

$$
\{f \in C(E): K \cap O(f) \neq \varnothing\},
$$

so that $f$ is in $L(K)$ if $K$ intersects the orthant of $V(E)$ corresponding to $f$. A subset $L$ of $C(E)$ will be called realizable if there is a convex set $K$ with $L=L(K)$. The realizable sets have in common a rather strong property, "lopsidedness," which will be described in §2. Examples showing how lopsided sets may arise in other settings will be given.

In $\S \S 3$ and 4 , two other descriptions of lopsided sets will be given. That of $\S 4$ is used in $\$ 5$ to show how lopsided sets may be derived from oriented matroids. Also in $\S 5$, the property of lopsidedness is used to give a new description of the simple oriented matroids. (For a discussion of oriented matroids, see Folkman and Lawrence [4], or Bland and Las Vergnas [1].)

Finally, $\$ 6$ gives an example of a lopsided set which is not realizable. This set is a subset of an 8-dimensional cube. It is not known whether this is the smallest dimension for such a set. 
2. Definition and examples of lopsided sets. A set $L \subset C(E)$ will be called lopsided if, whenever $A$ and $B$ are subsets of $E$ with $A \cup B=E$ and $A \cap B=\varnothing$, either:

(1) There is a function $\alpha: A \rightarrow\{-1,1\}$ such that all the functions $f$ in $C(E)$ which agree with $\alpha$ on $A$ are in $L$; or

(2) There is a function $\beta: B \rightarrow\{-1,1\}$ such that none of the functions in $C(E)$ which agree with $\beta$ on $B$ are in $L$.

For notational convenience we make use of the existence of the empty function $\eta: \varnothing \rightarrow\{-1,1\}$, whose domain is the empty set. This function is the sole member of $C(\varnothing)$, so that $|C(E)|=2^{|E|}$, for any set $E$. We will use $1_{x}$ to denote the function $1_{x}:\{x\} \rightarrow\{-1,1\}$ with $1_{x}(x)=1$. If $A$ and $B$ are disjoint sets, $\mathscr{F} \subset V(A)$, and $\mathcal{G} \subset V(B)$, then $\mathscr{F} \times \mathcal{G}$ is the set of functions $f$ in $V(A \cup B)$ which agree with a function in $\mathscr{F}$ on $A$ and with one of $\mathcal{G}$ on $B$. Then $V(A \cup B)=V(A) \times V(B)$. If $\eta$ is the empty function, $\{\eta\} \times V(B)=V(B)$.

The set $L \subset C(E)$ is lopsided if and only if, whenever $A$ and $B$ are complementary subsets of $E$, either:

(1) There is an element $\alpha$ of $C(A)$ with $\{\alpha\} \times C(B) \subset L$; or

(2) There is an element $\beta$ of $C(B)$ with $C(A) \times\{\beta\} \subset C(E) \sim L$.

Clearly, not both conditions can hold at once, for this would imply that $\{\alpha\} \times\{\beta\}$ is contained in $L$, as well as in $C(E) \sim L$. Also, it's clear that $L$ is lopsided if and only if $C(E) \sim L$ is lopsided.

This definition resembles that of a "blocking system." (See Edmonds and Fulkerson, [3].) In fact, we can make use of this notion here. Suppose $\Re$ and $\mathcal{S}$ are collections of subsets of the set $E$. Then the pair $(\Re, \mathcal{S})$ is a blocking system if, whenever $A$ and $B$ are subsets of $E$ with $A \cup B=E$ and $A \cap B=\varnothing$, either $A$ contains some member of $\mathcal{R}$ or $B$ contains some member of $\mathcal{S}$, but not both.

Now, consider a subset $L$ of $C(E)$.

Consider the collections:

$$
\begin{aligned}
& \Re=\{A \subset E: \text { there is } \alpha \in C(A) \text { with }\{\alpha\} \times C(B) \subset L\} ; \\
& \mathcal{S}=\{B \subset E: \text { there is } \beta \in C(B) \text { with } C(A) \times\{\beta\} \subset C(E) \sim L\} .
\end{aligned}
$$

Clearly $L$ is lopsided if and only if the pair $(\Re, S)$ is a blocking system.

We shall see that the realizable sets all possess the property of lopsidedness.

THEOREM 1. If $L=L(K)$ is realizable, then $L$ is lopsided.

Suppose $E=A \cup B$, where $A \cap B=\varnothing$. We may assume $A$ and $B$ are non-empty. 
It is convenient here to view $V(A)$ and the subspace $V(A) \times\{0\}$ of $V(E)$ as being identified in the obvious way.

Consider the map $\pi: V(E) \rightarrow V(B)$ which takes the function $f$ on $E$ to its restriction to $B$. The kernel of this map is $V(A)$, so $K \cap V(A) \neq \varnothing$ if and only if $\pi(K)$ contains the origin.

Either $K \cap V(A) \neq \varnothing$, and it follows that (1) holds for $L(K)$, or $\pi(K)$ doesn't contain the origin, in which case $\pi(K)$, being convex, must miss an entire orthant of $V(B)$, and (2) holds.

Now we consider some examples of lopsided sets. All of the examples in this section are realizable. There are lopsided sets which are not realizable, however, as an example in $\$ 6$ will show.

Let $(E, \delta)$ be a simplicial complex, so that $E$ is a finite set and $\delta$ is a collection of subsets of $E$ such that if $T$ is in $\delta$ and $S \subset T$ then $S$ is in $\mathcal{S}$. For $S$ in $\mathcal{S}$, let $f_{S}$ be the function defined on $E$ with:

$$
f_{S}(x)= \begin{cases}-1 & \text { if } x \notin S \\ 1 & \text { if } x \in S .\end{cases}
$$

Then the set:

$$
L=\left\{f_{S}: S \in \mathcal{S}\right\}
$$

is a lopsided set. Indeed $L=L(K)$, where $K$ is the convex set of functions $f$ in $V(E)$ with:

(i) $f(x) \leq 0$ for each $x$ in $E$; and

(ii) $\{x: f(x)=0\}$ is in $\delta$.

Now consider a second class of examples. Let $G$ be a directed graph with no loops or multiple edges and such that, if there is an edge from $u$ to $v$, then there is not an edge from $v$ to $u$. Let $s$ and $t$ be distinct vertices of $G$. Let $E$ be a subset of the set of edges of $G$. For a function $f$ in $C(E)$, let $G(f)$ be the graph obtained from $G$ by replacing each edge $e$ of $E$ for which $f(e)=-1$ by the edge connecting the same pair of vertices, but oriented in the opposite direction. For instance, if $f(e)=1$ for each $e$ in $E$, then $G(f)=G$. Let $L$ consist of all functions $f$ in $C(E)$ for which $G(f)$ contains the edges of a path from $s$ to $t$. It is possible to verify that $L$ is a lopsided subset of $C(E)$. Indeed, a more general result will be proven in $\$ 5$ (Theorem 7).

Finally, let $E$ be a finite set of lines in the plane, $R^{2}$, and suppose no three of these lines have a point in common. Let $K$ be a convex, open set in the plane. For each line $e$ in $E$, let one half-plane determined by $e$ be designated as its "positive" side, the other as its "negative" side. 
The lines in $E$ cut $K$ into open regions, and corresponding to each such region $r$ there is a function $f$ in $C(E)$ with:

$$
f(e)= \begin{cases}1 & \text { if the region } r \text { is on the positive side of } e \\ -1 & \text { otherwise. }\end{cases}
$$

That the set $L$ of such functions is lopsided can be seen from Theorem 1. For each line $E$, let $A_{e}$ be an affine functional on $R^{2}$ which is zero on $e$, positive on the positive side of $e$, and negative on the negative side of $e$. Let $A$ be the mapping that takes the point $p$ of $R^{2}$ to the function $f$ of $V(E)$ with $f(e)=A_{e}(p)$, for each $e$ in $E$. Let $K^{\prime}$ be the image of $K$ under this mapping. It is easy to verify that $L=L\left(K^{\prime}\right)$.

We now come to a theorem which describes simple ways to obtain new lopsided sets from given ones.

THEOREM 2. Suppose $L$ is a lopsided subset of $C(E)$. Then:

(i) $C(E) \sim L$ is lopsided;

(ii) If $F$ is a non-empty face of the cube, then $F \cap L$ is lopsided;

(iii) If $S$ is a subset of $E$ and $\pi: V(E) \rightarrow V(S)$ is the restriction of $f$ to $S$, then the image $\pi(L)$ of $L$ is a lopsided subset of $C(S)$;

(iv) If, also, $L^{\prime}$ is a lopsided subset of $C\left(E^{\prime}\right)$, where $E^{\prime}$ is a set with $E^{\prime} \cap E=\varnothing$, then $L \times L^{\prime}$ is a lopsided subset of $C(E) \times C\left(E^{\prime}\right)$.

We have already noted the validity of (i). Also, (iv) will be left to the reader.

For (ii), it is obvious that it suffices to prove this in the case that $F$ is actually a facet of the cube. Then there is an $e$ in $E$ and a number $\varepsilon$, either 1 or -1 , such that $F$ is the set of functions $f$ in the cube with $f(e)=\varepsilon$. We will suppose that $F \cap L$ is not lopsided, and obtain a contradiction.

Since $F \cap L$ is not lopsided, there is a partition of $E$ into two sets $A$ and $B$ such that: and

(1) There is no $\alpha$ in $C(A)$ with $\{\alpha\} \times C(B) \subset L \cap F$;

(2) There is no $\beta$ in $C(B)$ with $C(A) \times\{\beta\} \subset C(E) \sim(L \cap F)$. Note that $e$ must be in $A$, since otherwise a function $\beta$ in $C(B)$ with $\beta(e)=-\varepsilon$ would contradict (2). Note, also, that (1) implies:

(1') There is no $\alpha$ in $C(A \sim\{e\})$ with $\{\alpha\} \times C(B \cup\{e\}) \subset L$. Since $L$ is lopsided, $\left(1^{\prime}\right)$ implies that there is a function $\beta^{\prime}$ in $C(B \cup\{e\})$ with $C(A \sim\{e\}) \times\left\{\beta^{\prime}\right\} \subset C(E) \sim L$. By (2), we must have $\beta^{\prime}(e)=-\varepsilon$. Similarly, (2) implies that there is $\alpha^{\prime}$ in $C(A)$ with $\left\{\alpha^{\prime}\right\} \times C(B) \subset L$. By (1), we must have $\alpha^{\prime}(e)=-\varepsilon$. 
Consider the function $f$ with:

$$
f(x)= \begin{cases}-\varepsilon & x=e \\ \alpha^{\prime}(x) & x \in A \sim\{e\} \\ \beta^{\prime}(x) & x \in B\end{cases}
$$

This function agrees with $\alpha^{\prime}$ on $A$, so it must be in $L$; on the other hand, it agrees with $\beta^{\prime}$ on $B \cup\{e\}$, so it cannot be in $L$. Clearly, both conclusions cannot hold.

To prove (iii), it is clear that we need only consider sets $S$ with $|S|=|E|-1$. Let $\{e\}=E \sim S$. Now suppose $S=A \cup B$ and $A \cap B=$ $\varnothing$. Since $L$ is lopsided, one of the following must hold.

(1) There is a function $\alpha$ on $A \cup\{e\}$ such that all the functions agreeing with $\alpha$ on $A \cup\{e\}$ are in $L$.

In this case, if $f$ is any function in $C(S)$ which agrees with $\alpha$ on $A$, then $f=\pi\left(f^{\prime}\right)$ is in $\pi(L)$, where:

$$
f^{\prime}(x)= \begin{cases}f(x) & x \in S \\ \alpha(e) & x=e .\end{cases}
$$

(2) There is a function $\beta$ on $B$ such that none of the functions in $C(E)$ which agree with $\beta$ on $B$ are in $L$.

In this case, if the function $f$ in $C(S)$ agrees with $\beta$ on $B$, then any function $f^{\prime}$ with $\pi\left(f^{\prime}\right)=f$ also agrees with $\beta$ on $B$, and is not in $L$. Therefore $f$ is not in $\pi(L)$.

It follows that $\pi(L)$ is lopsided.

Next we consider analogous properties of realizable sets.

Now, if $L=L(K)$ is a realizable set, then there is a polytope $K^{\prime}$ with $L=L\left(K^{\prime}\right)$. Indeed, if for each $f$ in $L$ we pick a point $f^{\prime}$ in $O(f) \cap K$, then the convex hull of these functions $f^{\prime}$ will do for $K^{\prime}$.

Identifying $V(E)$ with $R^{n}$ (where $n=|E|$ ), we can find an $m \times n$ matrix $A$ and a vector $b$ in $R^{n}$ with:

$$
K^{\prime}=\{x: A x \leq b\} .
$$

It is easily proven, utilizing Farkas' Lemma, that the set:

$$
\bar{K}=\left\{A^{T} y: y \in R^{m} \text { with } y \geq 0, y^{T} b<0\right\}
$$

intersects precisely those closed orthants which $K^{\prime}$ misses; i.e., $L(\bar{K})=$ $C(E) \sim L\left(K^{\prime}\right)$. We see that (1) holds for the realizable sets, as well as for the lopsided sets.

The other three parts of Theorem 2 can easily be verified for realizable sets. We leave this to the reader. 
3. Symmetry and lopsidedness. This section provides another characterization of the lopsided sets. This characterization gives some justification for the term "lopsided."

THEOREM 3. Suppose $L$ is a lopsided subset of $C(E)$. Suppose, further, that whenever $f$ is in $L$, the vertex antipodal to $f$ on the cube, $-f$, is also in $L$. Then either $L=\varnothing$ or $L=C(E)$.

Suppose, on the contrary, that $L$ is lopsided, symmetrical with respect to the antipodal mapping, and neither $\varnothing$ nor $C(E)$. Let $S$ and $T$ be complementary subsets of $E$, with $S$ of cardinality as small as possible, for which there is a function $\gamma$ in $C(S)$ with $\{\gamma\} \times C(T) \subset L$. Then $S$ is not empty. Let $x$ be an element of $S$. There is no function $\alpha$ in $C(S \sim\{x\})$ such that $\{\alpha\} \times C(T \cup\{x\}) \subset L$. Therefore, there must be a function $\beta$ in $C(T \cup\{x\})$ such that $C(S \sim\{x\}) \times\{\beta\} \subset C(E) \sim L$. Then $C(S \sim\{x\})$ $\times\{-\beta\} \subset C(E) \sim L$, as well, since $L$ is symmetric. However, one of these sets intersects $\{\gamma\} \times C(T)$, and we have a contradiction.

Suppose $L$ is a subset of the vertex-set of a face $F$ of the cube. Since $F$, itself, is a cube, we may inquire whether or not $L$ is lopsided with respect to $F$, the notion of lopsidedness depending only on the combinatorial structure of the cube. We will see that $L$ is lopsided with respect to $F$ if and only if it is lopsided with respect to the larger cube.

Let $S$ and $T$ be complementary subsets of $E$ and $\gamma$ an element of $C(S)$, such that $\{\gamma\} \times C(T)$ is the vertex-set of $F$. The subset $L$ of $\{\gamma\} \times C(T)$ is lopsided with respect to $F$ if, for any complementary subsets $A$ and $B$ of $T$, either:

(1) There is a function $\alpha$ in $C(A)$ with

$$
\{\gamma\} \times\{\alpha\} \times C(B) \subset L ;
$$

or (2) There is a function $\beta$ in $C(B)$ with

$$
\{\gamma\} \times C(A) \times\{\beta\} \subset C(E) \sim L .
$$

Since $L \subset\{\gamma\} \times C(T)$, these two conditions are equivalent to the following, if we let $A^{\prime}=A \cup S$ and $B^{\prime}=B$ :

(1') There is a function $\alpha^{\prime}$ in $C\left(A^{\prime}\right)$ with

$$
\left\{\alpha^{\prime}\right\} \times C\left(B^{\prime}\right) \subset L
$$

(2') There is a function $\beta^{\prime}$ in $C\left(B^{\prime}\right)$ with

$$
C\left(A^{\prime}\right) \times\left\{\beta^{\prime}\right\} \subset C(E) \sim L .
$$

Since $\left(1^{\prime}\right)$ and $\left(2^{\prime}\right)$ must hold for any set lopsided with respect to the larger cube, any such set is lopsided with respect to $F$. On the other hand, 
suppose $L$ is lopsided with respect to $F$, and $A^{\prime}$ and $B^{\prime}$ are complementary subsets of $E$. If $B^{\prime} \cap S \neq \varnothing$, then (2') holds, with $\beta^{\prime}$ any function which does not agree with $\gamma$ on $B^{\prime} \cap S$. If $B^{\prime} \cap S=\varnothing$, pick complementary subsets $A$ and $B$ of $T$ with $A^{\prime}=A \cup S$ and $B=B^{\prime}$. Since $L$ is lopsided with respect to $F,(1)$ or (2) (and, therefore, $\left(1^{\prime}\right)$ or $\left(2^{\prime}\right)$ ) must hold. It follows that $L$ is lopsided with respect to the larger cube, as well.

Now, if $L$ is lopsided, then, for each face $F$ of the cube, $L \cap F$ is lopsided. It follows if $L \cap F$ is symmetric with respect to the antipodal mapping for $F$, then $L \cap F$ must either be empty or contain all the vertices of $F$. Indeed, this condition characterizes the lopsided sets, as we shall see. Let us (temporarily) call a subset $L$ of $C(E)$ totally asymmetric if, whenever $F$ is a face of the cube and $L \cap F$ is closed under the antipodal mapping for $F$, either $L \cap F$ is empty or consists of all the vertices of $F$. We have seen that any lopsided set is totally asymmetric. Soon we shall see that any totally asymmetric set is lopsided. First, we prove four lemmas.

It is convenient here to consider the graph $G$ whose vertices and edges are the vertices and edges of the cube. $C(E)$ is the vertex-set of $G$. Two functions $f$ and $g$ are adjacent if they agree on all but one element of $E$.

LEMMA 1. Let $L \subset C(E)$ be a totally asymmetric set. Then the subgraph of $G$ induced by $L$ is connected.

Suppose not. Let $H$ be the subgraph induced by $L$. For vertices $h$ and $k$ of $C(E)$, let $d(h, k)$ be the Hamming distance between $h$ and $k$,

$$
d(h, k)=|\{t \in E: h(t) \neq k(t)\}| .
$$

Let $f$ and $g$ be vertices of $C(E)$ in different components of $H$ and such that $d(f, g)$ is as small as possible. Then, if $F$ is the smallest face containing $f$ and $g, F \cap L=\{f, g\}$ is symmetric but neither empty nor all the vertices of $F$. This cannot be the case, since $L$ is totally asymmetric.

It follows that the subgraph of $G$ induced by $L$ is connected. In fact, since, if $F$ is any face of the cube, $L \cap F$ is also lopsided, it follows that the subgraph induced by $L \cap F$ is connected. For a characterization of subgraphs of the $n$-cube which have this strong property, see Djokovic [2].

Suppose $x$ is in $E$, and $S=E \sim\{x\}$. Let $\pi$ be the function from $V(E)$ to $V(S)$ mapping a function in $V(E)$ to its restriction. Recall that $1_{x}:\{x\} \rightarrow\{-1,1\}$ is the function with $1_{x}(x)=1$.

LemMa 2. Suppose $L$ is a totally asymmetric subset of $C(E)$. If $\pi(L)=C(S)$, then $L$ contains all of $\left\{1_{x}\right\} \times C(S)$ or all of $\left\{-1_{x}\right\} \times$ $C(S)$. 
Suppose not. Choose $f$ and $g$ in $C(E) \sim L$ such that $f(x)=1$, $g(x)=-1$, with $d(f, g)$ as small as possible (where $d$ is Hamming distance, as in the proof of the preceding lemma). Then $f$ and $g$ are not adjacent since $\pi(L)=C(S)$. If $F$ is the smallest face containing $f$ and $g$, then $F \cap L$ is neither empty nor all the vertex set of $F$. This cannot be the case, since $L$ is totally asymmetric.

LEMMA 3. Let $L$ be a totally asymmetric subset of $C(E), S$ a subset of $E$, and $\pi$ the restriction-map of $V(E)$ to $V(S)$. Then $\pi(L)$ is a totally asymmetric subset of $C(S)$.

Suppose this is not correct. Let $E$ be a set with as few elements as possible so that there is a totally asymmetric subset $L$ of $C(E)$ and a subset $S$ of $E$ with $\pi(L)$ not totally asymmetric. We may assume that $|S|=|E|-1$, since if $S^{\prime} \supset S$ and $\left|S^{\prime}\right|=|E|-1$, the projection of $L$ to $C\left(S^{\prime}\right)$ must already fail to be totally asymmetric, by the minimality of $|E|$. Also it is clear that if $F$ is a proper face of the cube in $V(S)$ then $\pi(L) \cap F$ is totally asymmetric, since, if $\bar{F}$ is the inverse-image of $F$ under $\pi$, so that $\bar{F}=\pi^{-1}(F)$, then $L \cap \bar{F}$ is a totally asymmetric subset of $\bar{F}$ which projects to $\pi(L) \cap F$, and the dimension of $\bar{F}$ is smaller than $|E|$. It follows that $\pi(L)$ itself will be closed under the antipodal mapping for $C(S)$.

Suppose $y$ is in $S$. Let $T=E \sim\{y\}$, and let $\pi^{\prime}$ be the restriction-map of $V(E)$ to $V(T)$. We will show that $\pi^{\prime}(L)$ is closed under the antipodal mapping for $C(T)$. Clearly, we need only show that $\pi^{\prime}(L)$ is not totally asymmetric, by the minimality of $|E|$. Suppose, on the other hand, that $\pi^{\prime}(L)$ is totally asymmetric. Consider the subset $\pi\left(\pi^{\prime}(L)\right)$ of $C(S \cap T)$. $\pi\left(\pi^{\prime}(L)\right)=\pi^{\prime}(\pi(L))$, so the set is closed under the antipodal mapping for $C(S \cap T)$. It is not empty, since $L$ is not. If $\pi^{\prime}(L)$ is totally asymmetric, $\pi\left(\pi^{\prime}(L)\right)$ is, too (utilizing the minimality of $|E|$ ), so $\pi\left(\pi^{\prime}(L)\right)=$ $C(S \cap T)$.

Now, let $\{x\}=E \sim S$. By Lemma $2, \pi^{\prime}(L)$ must contain $\left\{1_{x}\right\} \times$ $C(S \cap T)$ or $\left\{-1_{x}\right\} \times C(S \cap T)$. Then $L$ must contain one of:

$$
\begin{gathered}
\left\{1_{y}\right\} \times\left\{1_{x}\right\} \times C(S \cap T) \\
\left\{-1_{y}\right\} \times\left\{1_{x}\right\} \times C(S \cap T) \\
\left\{1_{y}\right\} \times\left\{-1_{x}\right\} \times C(S \cap T)
\end{gathered}
$$

or

$$
\left\{-1_{y}\right\} \times\left\{-1_{x}\right\} \times C(S \cap T) .
$$


Finally, $\pi(L)$ contains all of $\left\{1_{y}\right\} \times C(S \cap T)$ or $\left\{-1_{y}\right\} \times$ $C(S \cap T)$; but, since $\pi(L)$ is symmetric, $\pi(L)$ is all of $C(S)$. This cannot be the case since $C(S)$ is totally asymmetric. Therefore, $\pi^{\prime}(L)$ is not totally asymmetric, and, as we have seen, must be closed under the antipodal mapping for $C(T)$.

Since $L$ is not symmetric, there is a vertex $f$ in $L$ such that $-f$ is not in $L$. Now, suppose $g$ is adjacent to $-f$, so that $-f$ and $g$ differ on only one element of $E$, say $u$. Let $U=E \sim\{u\}$. Let $\pi^{\prime \prime}$ be the restriction-mapping of $V(E)$ to $V(U)$. We have seen that $\pi^{\prime \prime}(L)$ is a symmetric subset of $C(U)$. Since $\pi^{\prime \prime}(f)$ is in $\pi^{\prime \prime}(L), \pi^{\prime \prime}(-f)=\pi^{\prime \prime}(g)$ must be in $\pi^{\prime \prime}(L)$. It follows that $g$ must be in $L$. That is, all the vertices of $C(E)$ adjacent to $-f$ are in $L$.

Now, $L^{\prime}=C(E) \sim L$ is also totally asymmetric, $-f$ is in $L^{\prime}$ and is adjacent to no other vertex in $L^{\prime}$. It follows by Lemma 1 that $L^{\prime}=\{-f\}$, so that $L=C(E) \sim\{-f\}$. Then $\pi(L)=C(S)$, a totally asymmetric set, and we have a contradiction.

Utilizing Lemma 3, we can extend Lemma 2 somewhat.

Lemma 4. Suppose $E$ is the disjoint union of the sets $R, S$, and $T$. Suppose $L$ is a totally asymmetric subset of $C(E), \pi$ is the restriction-mapping of $C(E)$ to $C(R \cup S), \gamma \in C(R)$, and $\{\gamma\} \times C(S) \subset \pi(L)$. Then there is an element $\beta$ of $C(T)$ with $\{\gamma\} \times C(S) \times\{\beta\} \subset L$.

We proceed by induction on $|T|$. If $|T|=1$, the result is an easy consequence of Lemma 2. Suppose $|T|=n>1$, and that the result holds when the set has cardinality less than $n$.

Let $x$ be an element of $T$. Let $T^{\prime}=T \sim\{x\}$. Let $\pi^{\prime}: V(E) \rightarrow$ $V(R \cup S \cup\{x\})$ and $\pi^{\prime \prime}: V(R \cup S \cup\{x\}) \rightarrow V(R \cup S)$ be the restriction-mappings, so that $\pi=\pi^{\prime \prime} \circ \pi^{\prime}$. Now, $\pi^{\prime}(L)$ is a totally asymmetric subset of $C(R \cup S \cup\{x\})$, by Lemma 3, and

$$
\pi^{\prime \prime}\left(\pi^{\prime}(L)\right)=\pi(L) \supset\{\gamma\} \times C(S) .
$$

The inductive assumption yields a function $\beta^{\prime \prime} \in C(\{x\})$ with $\{\gamma\} \times$ $C(S) \times\left\{\beta^{\prime \prime}\right\} \subset \pi^{\prime}(L)$. Next, since $\left|T^{\prime}\right|<|T|$, there is a function $\beta^{\prime} \in$ $C\left(T^{\prime}\right)$ with $\{\gamma\} \times C(S) \times\left\{\beta^{\prime \prime}\right\} \times\left\{\beta^{\prime}\right\} \subset L$. Clearly, the function $\beta$ in $C(T)$ which agrees with $\beta^{\prime \prime}$ on $x$ and with $\beta^{\prime}$ on $T^{\prime}$ is the function required.

THEOREM 4. Suppose $L$ is a totally asymmetric set. Then $L$ is lopsided.

Suppose $A$ and $B$ are disjoint subsets of $E$. Let $\pi: V(E) \rightarrow V(B)$ be the restriction mapping. Then either: (1) $C(B) \subset \pi(L)$, so that (by 
Lemma 4 with $R=\varnothing, S=B$, and $T=A)$ there is $\alpha \in C(A)$ with $\{\alpha\} \times C(B) \subset L$; or (2) There is $\beta \in C(B)$ with $\beta \notin \pi(L)$; i.e., so that $C(A) \times\{\beta\} \subset C(E) \sim L$.

4. Route systems. Next, we characterize the lopsided sets in yet another way. This one will be useful in the next section, where we relate lopsided sets and oriented matroids.

We focus on the collection of maximal faces of the cube which miss $L$.

Let $\mathscr{F}$ denote the set of facets of the cube. $|\mathscr{F}|=2|E|$. If $F$ is an element of $\mathscr{F}$, let $F^{*}$ denote the (unique) facet having empty intersection with $F$.

If $\mathcal{G}$ is a subset of $\mathscr{F}$, we write the intersection $G$, of the facets in $\mathcal{G}$, as $G=\cap \mathcal{G}$.

If $\mathcal{G} \subset \mathscr{F}$, let $\mathcal{G}^{*}=\left\{G^{*} \mid G \in \mathcal{G}\right\}$. Note that, if $\mathcal{G} \subset \mathcal{F}$, then $\cap \mathcal{G}$ is a non-empty face of the cube if and only if $\mathcal{G} \cap \mathcal{G}^{*}=\varnothing$. This face is a vertex if, also $\mathcal{G} \cup \mathcal{G}^{*}=\mathscr{F}$.

Now, let $L$ be a lopsided subset of $C(E)$. Let $\Gamma$ denote the collection of subsets $\Re$ of $\mathscr{F}$, minimal with respect to set inclusion, such that:

(1) $\Re \cap \Re *=\varnothing$; and

(2) $L \cap(\cap \Re)=\varnothing$.

That is, $\Re \in \Gamma$ if and only if $\cap \Re$ is a non-empty face of the cube maximal among those which miss $L$. We call the elements of $\Gamma$ the routes corresponding to $L$, and $\Gamma$ is the route system.

To motivate this terminology consider an example arising from a directed graph $G$, as in $\$ 2$. Recall that $E$ is a set of edges of $G$. If $e \in E$ let $\hat{e}$ denote an edge connecting the same two vertices as $e$, but going the opposite direction. The facets of the cube correspond to the $2|E|$ directed edges in $E \cup\{\hat{e} \mid e \in E\}$. Let $L$ be the lopsided set arising as in Section 2. Let $\Gamma$ be the route system corresponding to the lopsided set $C(E) \sim L$. In this case, a route is simply a set of facets corresponding to the edges in a simple path from $s$ to $t$.

In this section we will characterize the route systems.

Suppose $A$ and $B$ are complemenary subsets of $E$, and $\alpha$ and $\alpha^{\prime}$ are in $C(A)$. If $F$ and $F^{\prime}$ are the faces of the cube in $V(E)$ whose vertex-sets are $\{\alpha\} \times C(B)$ and $\left\{\alpha^{\prime}\right\} \times C(B)$, then $F^{\prime}$ is a translate of $F$, and any pair of faces which are translates of one another arise in this way. Call $F$ and $F^{\prime}$ adjacent if $\alpha$ and $\alpha^{\prime}$ are adjacent vertices of $C(A)$. Note that $F$ and $F^{\prime}$ are adjacent if and only if they are disjoint facets of a face of the cube that contains them both.

We can state a stronger form of Lemma 1. 
Lemma 5. Suppose $G$ and $G^{\prime}$ are faces of the cube which miss the lopsided set $L$, and that $G^{\prime}$ is a translate of $G$. Then there is a sequence $G_{0}=G, G_{1}, \ldots, G_{k}=G^{\prime}$ of faces, translates of $G$, which miss $L$, such that $G_{i-1}$ and $G_{i}$ are adjacent, for $1 \leq i \leq k$. Furthermore, we may assume that each of these faces is contained in the smallest face of the cube which contains $G$ and $G^{\prime}$.

Pick complementary subsets $A$ and $B$ of $E$ and $\alpha, \alpha^{\prime} \in C(A)$ so that $\{\alpha\} \times C(B)$ and $\left\{\alpha^{\prime}\right\} \times C(B)$ are the vertex-sets of $G$ and $G^{\prime}$. Consider the restriction mapping $\pi: V(E) \rightarrow V(A)$. Since $G$ and $G^{\prime}$ miss $L, \alpha$ and $\alpha^{\prime}$ are both in the lopsided set $C(A) \sim \pi(L)$. There is a path from $\alpha$ to $\alpha^{\prime}$ in this set, $\alpha_{0}=\alpha, \alpha_{1}, \ldots, \alpha_{k}=\alpha^{\prime}$. For $0 \leq i \leq k$, let $G_{i}=\left\{\alpha_{i}\right\} \times C(B)$.

The following theorem gives a property of route systems.

THEOREM 5. If $\Re$ and $\delta$ are routes, corresponding to a lopsided set $L$, and $F \in \Re \cap \mathcal{S}^{*}$, then there is a route $\mathcal{T}$ with:

$$
\sigma \subset(\Re \cup S) \sim\left\{F, F^{*}\right\} .
$$

Let $\mathscr{Q}=\Re \cup\left(\delta \sim \Re^{*}\right)$ and $\mathscr{B}=\mathcal{S} \cup\left(\Re \sim \mathcal{S}^{*}\right)$. Let $G=\cap \mathbb{Q}$ and $G^{\prime}=\cap \mathscr{B}$. Then $G^{\prime}$ is a translate of $G$. $G \subset \cap \Re$ and $G^{\prime} \subset \cap \mathcal{S}$, so $G$ and $G^{\prime}$ each miss $L$. There is a path $G_{0}=G, G_{1}, \ldots, G_{k}=G^{\prime}$ as described in Lemma 5. Now $G \subset F$ and $G^{\prime} \subset F^{*}$. Each translate of $G$ is contained wholly in $F$ or in $F^{*}$. There must be a face $G_{i} \subset F$ with $G_{i+1} \subset F^{*}$. Let $H$ be the fact of the cube having $G_{\imath}$ and $G_{i+1}$ as facets. Let $\mathcal{C}=\{K \in \mathscr{F}$ : $H \subset K\}$.

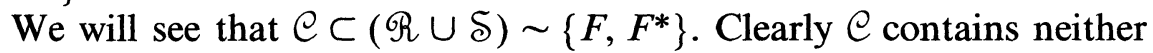
$F$ nor $F^{*}$. Since $\left.G_{i}=\cap(\mathcal{U} \cup F\}\right)$ is a translate of $G=\cap \mathbb{Q}, \bigodot \subset \mathbb{Q} \cup$ $\mathbb{Q}^{*} \subset(\Re \cup \mathcal{S}) \cup\left(\Re^{*} \cup \mathcal{S}^{*}\right)$. Since $H$ is on the smallest face of the cube containing $G$ and $G^{\prime}$ - that is, on $\cap\left(\left(\Re \sim \mathcal{S}^{*}\right) \cup\left(\mathcal{S} \sim \Re^{*}\right)\right)-\bigodot$ contains $\left(\Re \sim \mathcal{S}^{*}\right) \cup\left(\mathcal{S} \sim \Re^{*}\right)$. Since $\bigodot \cap \bigodot^{*}=\varnothing$, $\circlearrowright$ contains no element of $(\Re * \sim \mathcal{S}) \cup\left(\mathcal{S}^{*} \sim \Re\right)$. Then:

$$
\begin{aligned}
& \circlearrowright \subset\left((\Re \cup S) \cup\left(\Re * \cup S^{*}\right)\right) \sim\left((\Re * \sim S) \cup\left(\delta^{*} \sim \Re\right)\right) \\
& =\Re \cup \delta .
\end{aligned}
$$

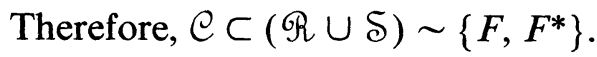

Since $H=\cap \mathcal{C}$ misses $L, \mathcal{C}$ contains the required route $\mathcal{T}$.

We will show that the conditions in Theorem 5 actually can be used to characterize the route systems. For what follows, suppose that $\Gamma$ is a 
collection of subsets of $\mathscr{F}$, no one of which contains another (a clutter), with:

(1) If $\Re \in \Gamma$ then $\Re \cap \Re^{*}=\varnothing$; and

(2) If $\Re, \delta \in \Gamma$ and $F \in \Re \cap \mathcal{S}^{*}$, then there is an element

$$
\mathcal{T} \text { of } \Gamma \text { with } \mathcal{T} \subset(\Re \cup \mathcal{S}) \sim\left\{F, F^{*}\right\} \text {. }
$$

If $f$ is in $C(E)$, let $\mathcal{H}(f)=\{F \in \mathscr{F}: f \in F\}$, so that $\{f\}=\cap \mathcal{H}(f)$. $F \in \mathcal{H}(f)$ if and only if $F^{*} \notin \mathcal{H C}(f)$. Let $L=\{f \in C(E)$ : $\mathcal{H}(f)$ contains no element of $\Gamma$. Note that $L$ consists of the vertices not lying in any face $\cap \Re$ for $\Re \in \Gamma$.

LEMMA 6. If $\mathscr{B}$ is a subset of $\mathscr{F}$ with $\mathscr{\Re} \cap \Re^{*}=\varnothing$, and if the face $\cap \Re$ misses $L$, then $\Re$ contains an element of $\Gamma$.

Suppose this is not the case. Choose a maximal subset $\mathscr{B}$ of $\mathscr{F}$ with:

(1) $\mathscr{B} \cap \Re^{*}=\varnothing$;

(2) $\cap \Re$ misses $L$; and

(3) $\mathscr{B}$ contains no element of $\Gamma$.

Clearly $\mathscr{B} \cup \mathscr{B}^{*} \neq \mathscr{F}$, for then, if $f$ is the vertex of $C(E)$ with $\{f\}=\cap \mathscr{B}$ (or, $\mathscr{B}=\mathscr{H}(f)$ ) we would have, from (3), $f \in L$. Let $F$ be an element of $\mathscr{F} \sim\left(\mathscr{\Re} \cup \Re^{*}\right)$. Then $\Re \cup\{F\}$ and $\Re \cup\left\{F^{*}\right\}$ satisfy (1) and (2), and each is larger than $\mathscr{B}$. Therefore there are elements $\mathscr{R}$ and $\mathcal{S}$ of $\Gamma$ with $\Re \subset \mathscr{B} \cup\{F\}$ and $\mathcal{S} \subset \mathfrak{B} \cup\left\{F^{*}\right\} . F$ must be in $\Re \cap \mathcal{S}^{*}$, since $\mathscr{B}$ satisfies (3). It follows that there is an element $\mathcal{T}$ of $\Gamma$ with $\mathcal{T} \subset(\Re \cup \mathcal{R}) \sim$ $\left\{F, F^{*}\right\} \subset \Re$, contradicting our assumption.

THEOREM 6. $L$ is a lopsided set and $\Gamma$ is its route system.

Let $\mathbb{Q}$ be a subset of $\mathscr{F}$ with $\mathscr{Q} \cap \mathbb{Q}^{*}=\varnothing$, so that $G=\cap \mathbb{Q}$ is a face of the cube, and suppose $L$ neither misses $G$ nor contains all the vertices of $G$. To show $L$ is lopsided, we need only show that $L \cap G$ is not closed under the antipodal mapping of $G$.

Let $\mathscr{B}^{\prime}$ be a set, of minimal cardinality, containing $\mathcal{A}$, with $\mathscr{B}^{\prime} \cap$ $\left(\Re^{\prime}\right)^{*}=\varnothing$ and $G^{\prime}=\cap \Re^{\prime}$ missing $L$. By Lemma 6, $\Re^{\prime}$ contains an element $\Re$ of $\Gamma$. Clearly, $\Re^{\prime} \sim Q \subset R$, by the minimality of $\left|\Re^{\prime}\right|$; and $\Re^{\prime} \sim$ $Q \neq \varnothing$.

Consider the set $\Re^{\prime \prime}=\mathscr{Q} \cup\left(\left(\Re^{\prime}\right)^{*} \sim \mathbb{Q}^{*}\right)$, which satisfies $\Re^{\prime \prime} \cap$ $\left(\Re^{\prime \prime}\right)^{*}=\varnothing$.

If $G^{\prime \prime}=\cap \Re^{\prime \prime}$ misses $L$, then $\Re^{\prime \prime}$ also contains an element, $\mathfrak{S}$, of $\Gamma$. Since $\left|\mathscr{B}^{\prime \prime}\right|=\left|\mathscr{B}^{\prime}\right|, \delta$ must contain $\mathscr{B}^{\prime \prime} \sim \mathbb{Q}=\left(\mathscr{B}^{\prime}\right)^{*} \sim \mathbb{Q}^{*}$. Then, let $F$ be an element of $\Re^{\prime} \sim \mathcal{Q} . F$ is in $\Re \cap \mathcal{S}^{*}$, so there is an element $\mathcal{T}$ of $\Gamma$ with $\sigma \subset(\Re \cup S) \sim\left\{F, F^{*}\right\}$. Let $\mathcal{T}=\mathcal{T} \cup \mathbb{Q}$. Then $\mathcal{C} \subset\left(\mathscr{B}^{\prime} \cup \mathscr{B}^{\prime \prime}\right) \sim\left\{F, F^{*}\right\}$ and $C \cap \bigodot^{*}=\varnothing$ so $\left|\mathcal{C}^{*}<\right| \mathscr{B}^{\prime} \mid$; but $\mathcal{C}$ contains $\mathscr{T}$, so $\cap \mathcal{C}$ misses $L$. This 
cannot be the case, since $\mathscr{B}^{\prime}$ was chosen with cardinality as small as possible. It follows that $G^{\prime \prime}$ does not miss $L$.

Since $G^{\prime \prime}$ is antipodal to $G^{\prime}$ on $G$, it follows that $L \cap G$ is not closed under the antipodal mapping of $G$. Thus $L$ is lopsided.

That $\Gamma$ is the route system for $L$ follows from Lemma 6.

5. Lopsided sets and oriented matroids. In this section we link the subject of lopsided sets to that of oriented matroids. The reader may wish to consult Folkman and Lawrence [4] or Bland and Las Vergnas [1] for an introduction to oriented matroids. However, the material needed from [4] will be reviewed here.

An oriented matroid is a triple $(\mathscr{F}, \Gamma, *)$, where ${ }^{*}$ is an involution on $\mathscr{F}$ (so that, if $F \in \mathscr{F}$, then $F^{*} \in \mathscr{F}, F^{*} \neq F$, and $\left(F^{*}\right)^{*}=F$ ), and $\Gamma$ is a clutter of subsets of $\mathscr{F}$, with:

(1) If $\Re \in \Gamma$ then $\Re^{*} \in \Gamma$, and $\Re \cap \Re^{*}=\varnothing$; and

(2) If $\Re, S \in \Gamma, F \in \Re \cap \mathfrak{S}^{*}$, and $\mathscr{R} \neq \mathfrak{S}^{*}$, then there is an element

$$
\mathcal{T} \text { of } \Gamma \text { with } \mathcal{T} \subset(\Re \cup \mathcal{S}) \sim\left\{F, F^{*}\right\} \text {. }
$$

If $\left(\mathscr{F}, \Gamma,{ }^{*}\right)$ is an oriented matroid then the following condition, apparently stronger than (2), holds:

$\left(2^{\prime}\right)$ If $\Re, \mathcal{S}$ are in $\Gamma, F \in \Re \cap \mathcal{S}^{*}$, and $G \in \Re \sim \mathcal{S}^{*}$, then there is an element $\mathcal{T}$ of $\Gamma$ with:

$$
G \in \mathcal{T} \subset(\Re \cup \delta) \sim\left\{F, F^{*}\right\} .
$$

The elements of $\Gamma$ are called the cycles of the oriented matroid. (For this improvement of terminology over [4], we are indebted to T. Zaslavsky.)

$\left(\mathscr{F}, \Gamma,{ }^{*}\right)$ is a simple oriented matroid if it is an oriented matroid, and there is an integer $k$ for which:

(1) If $\Re \in \Gamma$ then $|\Re|=k+1$; and

(2) If $\mathscr{C} \subset \mathcal{F}, \mathbb{Q}=\mathbb{Q}^{*}$, and $|\mathscr{Q}| \geq 2(k+1)$ then

$\mathbb{Q}$ contains an element $\Re$ of $\Gamma$.

The number $k$ is the rank of the simple oriented matroid.

Let $\left(\mathscr{F}, \Gamma,{ }^{*}\right)$ be an oriented matroid. Suppose $G \in \mathscr{F}$. Let $\mathscr{F}^{\prime}=\mathscr{F} \sim$ $\left\{G, G^{*}\right\}$ and let

$$
\Gamma^{\prime}=\left\{\Re \subset \mathscr{F}^{\prime}: \Re \cup\{G\} \text { is in } \Gamma\right\}
$$

THEOREM 7. $\left(\mathcal{F}^{\prime}, \Gamma^{\prime},{ }^{*}\right)$ is a route system.

If $\Re \in \Gamma^{\prime}$, then:

$$
\Re \cap \Re * \subset(\Re \cup\{G\}) \cap(\Re \cup\{G\})^{*}=\varnothing .
$$


Suppose $R$ and $\mathcal{S}$ are in $\Gamma^{\prime}$ and $F$ is in $R \cap \mathcal{S}^{*}$. Let $\Re_{0}=\Re \cup\{G\}$ and $\mathfrak{S}_{0}=\mathscr{S} \cup\{G\} . \mathscr{R}_{0}$ and $\mathcal{S}_{0}$ are in $\Gamma, F$ is in $\mathscr{R}_{0} \cap \mathcal{S}_{0}^{*}$, so there is a cycle $\mathscr{T}_{0}$ of $\Gamma$ with

$$
G \in \mathscr{T}_{0} \subset\left(\Re_{0} \cup \mathscr{S}_{0}\right) \sim\left\{F, F^{*}\right\} .
$$

Then $\mathcal{T}=\mathcal{T}_{0} \sim\{G\}$ is the element of $\Gamma^{\prime}$ we require.

Suppose $\left(\mathscr{F}, \Gamma,{ }^{*}\right)$ is a simple oriented matroid. Suppose $\mathscr{F}$ is the set of facets of the cube, and $F \cap F^{*}=\varnothing$, as in Section 4. Let $L$ consist of the elements $f$ in $C(E)$ such that $\mathcal{H}(f)$ contains no cycle.

THEOREM 8. $L$ is a symmetric subset of $C(E)$; if $G$ is a proper face of $C(E)$, then $G \cap L$ is lopsided.

That $L$ is a symmetric subset of $C(E)$ follows from the fact that, if $\Re \in \Gamma$, then $\Re^{*} \in \Gamma$.

For the other assertion, we need only show that if $G$ is a facet then $G \cap L$ is lopsided. Let $\mathscr{F}^{\prime}=\mathscr{F} \sim\left\{G, G^{*}\right\}$. Let $\Gamma^{\prime}$ denote the collection of subsets $\Re$ of $\mathscr{F}^{\prime}$, minimal with respect to set inclusion, such that $\mathscr{R} \cup\{G\}$ contains an element of $\Gamma$; i.e., such that either $R$ or $R \cup\{G\}$ is an element of $\Gamma$.

It is obvious that the vertex $f$ of $G$ is in $G \cap L$ if and only if $\left\{F \in \mathscr{F}^{\prime} \mid f \in F\right\}$ contains no element of $\Gamma^{\prime}$. To complete the proof, we need only show that $\left(\mathscr{F}^{\prime}, \Gamma^{\prime}, *\right)$ is a root system.

Suppose there is an element $R$ of $\Gamma \cap \Gamma^{\prime}$. Let $H$ be in $\Re$. Note that there must be an element 2 of $\Gamma$ in $\left(\left\{G, G^{*}\right\} \cup \Re \cup \Re^{*}\right) \sim\left\{H, H^{*}\right\}$ since the oriented matroid is simple. 2 must contain $G$ or $G^{*}$; if the latter, replace 2 by $2 *$.

Thus there exists an element $\mathcal{S}$ of $\Gamma$ with $G \in \mathcal{S} \subset\{G\} \cup \Re \cup \Re^{*}$, with $\left|S \cap \Re^{*}\right|$ as small as possible. $\left|\mathcal{S} \cap \Re^{*}\right|>0$, since otherwise $\mathcal{S} \sim$ $\{G\}$ would be an element of $\Gamma^{\prime}$ properly contained in $R$.

Let $F$ be an element of $\mathcal{S} \cap \Re^{*}$. Since $G \in \mathcal{S} \sim \Re^{*}$, there is a cycle $\mathscr{T} \in \Gamma$ with $G \in \mathcal{T} \subset(\mathcal{S} \cup \Re) \sim\left\{F, F^{*}\right\}$. Then $G \in \mathcal{T} \subset\{G\} \cup \Re \cup$ $\Re^{*}$. Also, $\mathscr{T} \cap \Re^{*} \subset\left(\mathcal{S} \cap \Re^{*}\right) \sim\{F\}$, so $\left|\mathcal{T} \cap \Re^{*}\right|<\left|\delta \cap \Re^{*}\right|$, contradicting the minimality of $\mathcal{S}$. It follows that $\Gamma \cap \Gamma^{\prime}=\varnothing$; i.e., $\Re \in \Gamma^{\prime}$ if and only if $\mathscr{R} \cup\{G\} \in \Gamma$. By Theorem $7,\left(\mathscr{F}^{\prime}, \Gamma^{\prime},{ }^{*}\right)$ is a route system.

Theorem 8, above, demonstrates that the set $L$ so arising from a simple oriented matroid has a rather remarkable property. Theorem 9 will show that any such set $L$ comes in this way from some simple oriented matroid.

We require the following lemma, which is also in [4]. 
LEMMA 7. Suppose $\left(\mathscr{F}, \Gamma,{ }^{*}\right)$ is an oriented matroid, $\Re$ and $\mathcal{S}$ are cycles, and $\Re \subset \mathcal{S} \cup \mathcal{S}^{*}$. Then $\Re=\mathcal{S}$ or $\Re=\mathcal{S}^{*}$.

Suppose, on the contrary, that there is a cycle $\delta$ for which we can find a cycle $\Re \subset \mathcal{S} \cup \mathcal{S}^{*}$ with $\Re \neq \mathcal{S}$ and $\Re \neq \mathcal{S}^{*}$. Let such a cycle $\Re$ be chosen with $\left|R \cap \mathcal{S}^{*}\right|$ as small as possible. $\left|\Re \cap \delta^{*}\right| \neq 0$, since otherwise $\Re \subset \mathcal{S}$. Let $F$ be an element of $\Re \cap \mathcal{S}^{*}$. Since $R \neq \mathcal{S}^{*}$, there is a cycle $\mathcal{C}$ contained in $(\Re \cup \delta) \sim\left\{F, F^{*}\right\}$. $\circlearrowright$ is neither $\delta$ nor $\mathcal{S}^{*}$, since it contains neither $F$ nor $F^{*}$;

$$
\bigodot \cap \varsigma^{*} \subset\left(\Re \cup S^{*}\right) \sim\{F\},
$$

so $\left|\bigodot \cap S^{*}\right|<\left|\Re \cap \mathfrak{S}^{*}\right|$. This cannot be the case, so there is no such cycle $\mathcal{S}$, and the lemma is established.

THEOREM 9. Suppose $L$ is a symmetric subset of $C(E)$, and that for each facet $G$ of the cube, $G \cap L$ is lopsided. Let $\Gamma$ consist of the minimal sets $\Re \subset \mathcal{F}$ with:

(1) $\Re \cap \Re *=\varnothing$; and

(2) $\cap \Re$ misses $L$.

Then $\left(\mathscr{F}, \Gamma,{ }^{*}\right)$ is a simple oriented matroid.

We show, first, that $\left(\mathscr{F}, \Gamma,{ }^{*}\right)$ is an oriented matroid. Clearly, if $\Re \in \Gamma$ then $\Re^{*} \in \Gamma$, and $\Re \cap \Re^{*}=\varnothing$. Suppose $\Re$ and $\delta$ are in $\Gamma, F \in \Re \cap \mathcal{S}^{*}$ and $\Re \neq S^{*}$. We must show that there is an element $\mathcal{T}$ of $\Gamma$ with $\mathcal{T} \subset(\Re \cup S) \sim\left\{F, F^{*}\right\}$.

Since $\Re \neq \mathcal{S}^{*}$, there is an element $G$ in $\Re \sim \mathcal{S}^{*}$. Let $\mathscr{F}^{\prime}=\mathscr{F} \sim\left\{G, G^{*}\right\}$, and let $\Gamma^{\prime}$ consist of the minimal sets $\mathscr{\mathcal { F } ^ { \prime }}$ with $\mathscr{\mathscr { F } ^ { \prime }} \cap \mathrm{W}^{*}=\varnothing$ and $\cap(\mathscr{W} \cup\{G\})$ missing $L$. Since $L \cap G$ is lopsided, $\left(\mathscr{F}^{\prime}, \Gamma^{\prime},{ }^{*}\right)$ is a route system. $\Re^{\prime}=\Re \sim\{G\}$ is in $\Gamma^{\prime}$, and there is an element $\mathcal{S}^{\prime}$ of $\Gamma^{\prime}$ contained in $\mathcal{S} \sim\{G\}$. If $F^{*} \notin \mathcal{S}^{\prime}$ then $\mathcal{S}^{\prime} \cup\{G\}$ is the required element of $\Gamma$. If $F^{*} \in \mathcal{S}^{\prime}$, there is an element $\mathcal{T}^{\prime}$ of $\Gamma^{\prime}$ with $\mathcal{T}^{\prime} \subset\left(\mathcal{R}^{\prime} \cup \mathcal{S}^{\prime}\right) \sim\left\{F, F^{*}\right\}$, and $\mathcal{T}^{\prime}$ or $\mathcal{T}^{\prime} \cup\{G\}$ is the required element of $\Gamma$.

Next we show that if there exists $\Re \in \Gamma$ with $|\Re|=k+1$, and if $\mathbb{Q} \subset \mathscr{F}$ with $\mathbb{Q}=\mathbb{Q}^{*}$ and $|\mathscr{Q}|=2(k+1)$, then there is an element of $\Gamma$ contained in $\mathbb{Q}$.

Suppose there is no such cycle. Pick $\mathscr{K} \in \Gamma$ with $|\mathscr{K}| \leq k+1$ and $|\mathscr{K} \sim \mathscr{Q}|$ as small as possible. Since $\mathscr{K}$ cannot be contained in $\mathbb{Q}$, $|\mathscr{K} \sim \mathscr{Q}|>0$. Choose $F \in \mathscr{K} \sim \mathcal{Q}$, and $G \in \mathbb{Q} \sim\left(\mathscr{K} \cup \mathscr{K}^{*}\right)$. Let $\mathscr{F}^{\prime}=\mathscr{F} \sim$ $\left\{G, G^{*}\right\}$. Let $\Gamma^{\prime}$ consist of the minimal sets $\mathscr{} \subset \mathscr{F}^{\prime}$ with $\mathscr{W} \cap \mathscr{W}^{*}=$ $\varnothing$ and $\cap(\mathscr{\mho} \cup\{G\})$ missing $L$. Since $L \cap G$ is lopsided, $\left(\mathscr{F}^{\prime}, \Gamma^{\prime}, *\right)$ is a route system. 
Since $\cap \mathscr{K}$ and $\cap \mathscr{K}^{*}$ miss $L$, there are elements $\Re$ and $\delta$ of $\Gamma^{\prime}$ with $\Re \cap \mathcal{K}$ and $\delta \subset \mathcal{K}^{*}$.

One of $R$ and $R \cup\{G\}$ is in $\Gamma$. Call this $R^{\prime}$. If $F \notin \Re$ then $\Re^{\prime} \subset(\mathscr{K} \cup\{G\}) \sim\{F\}$, so $\Re^{\prime} \sim \mathscr{Q} \subset \mathscr{K} \sim(\mathscr{Q} \cup\{F\}) ; \quad\left|\Re^{\prime}\right| \leq|\mathcal{K}| \leq$ $k+1$ and $\left|\Re^{\prime} \sim \mathscr{Q}\right|<|\mathcal{K} \sim \mathscr{Q}|$. This cannot be the case, since $\mathscr{K}$ is as small as possible, so $F \in R$. Similarly, $F^{*} \in \mathcal{S}$. Then there is $\mathcal{T} \in \Gamma^{\prime}$ with $\mathcal{T} \subset(\Re \cup \mathcal{S}) \sim\left\{F, F^{*}\right\}$. Either $\mathcal{T} \in \Gamma$ of $\mathcal{T} \cup\{G\} \in \Gamma$. Call this element of $\Gamma, \mathcal{T}^{\prime}$. Then:

$$
\mathcal{T}^{\prime} \subset\left(\mathscr{K} \cup \mathscr{K}^{*} \cup\{G\}\right) \sim\left\{F, F^{*}\right\}
$$

so:

$$
\mathcal{T}^{\prime} \sim \mathbb{Q} \subset\left(\mathscr{K} \cup \mathscr{K}^{*}\right) \sim\left(\mathbb{Q} \cup \mathbb{Q}^{*} \cup\left\{F, F^{*}\right\}\right) .
$$

Since $\mathcal{T}^{\prime} \cap\left(\mathcal{T}^{\prime}\right)^{*}=\varnothing$,

$$
\left|\mathcal{T}^{\prime}\right| \leq|\mathfrak{K}| \leq k+1 \text { and }\left|\mathcal{T}^{\prime} \sim \mathscr{Q}\right|<|\mathscr{K} \sim \mathbb{Q}| .
$$

We have a contradiction. Therefore, there must be an element of $\Gamma$ contained in $Q$.

Finally, we show that any two elements of $\Gamma$ must have the same cardinality. Suppose $R, \delta \in \Gamma$. We may suppose $|\Re| \leq|\delta|$. Let $\mathscr{Q}$ be a subset of $\delta \cup \mathcal{S}^{*}$ with $\mathscr{Q}=\mathbb{Q}^{*}$ and $|\mathscr{Q}|=2|\Re|$. As we have seen, there is an element $\mathcal{T}$ of $\Gamma$ with $\mathcal{T} \subset \mathbb{Q}$. Then $\mathcal{T} \subset \mathcal{S} \cup \mathcal{S}^{*}$ so, by Lemma $7, \mathcal{T}=\mathcal{S}$ or $\mathcal{T}=\mathcal{S}^{*}$. It follows that $\mathcal{S} \cup \mathcal{S}^{*}=\mathscr{Q}$ and, since $\mathcal{S} \cap \mathcal{S}^{*}=\varnothing,|\mathfrak{S}|=$ $\frac{1}{2}|\mathbb{Q}|=|\Re|$.

6. A non-realizable lopsided set. In this section we obtain an example of a lopsided set which is not realizable by considering a certain "non-stretchable arrangement of pseudolines" with another special property.

A pseudoline is a closed subset of the plane which is homeomorphic to the real line. An arrangement of pseudolines is a finite set of pseudolines in the plane, each pair of which have exactly one point of intersection, where they cross. Two such arrangements are equivalent if there is a homeomorphism of the plane which takes the pseudolines of one to those of the other. Finally, an arrangement of pseudolines is stretchable if it equivalent to an arrangement of genuine lines.

Levi [6] was the first to note the existence of arrangements of pseudolines that are not stretchable. Ringel [7] found an example of a non-stretchable arrangement which is simple; i.e., such that no three of the pseudolines have a point in common. The arrangement pictured in The Figure is taken from Grünbaum [5] (page 42), where one may find a fuller history and description of the subject. 
The importance of such arrangements here lies in the fact that they yield examples of lopsided sets, just as arrangements of lines did in \$2. Suppose $E$ is an arrangement of pseudolines. For each pseudoline $p$ in $E$, designate one of the closed "half-planes" determined by $p$ as its positive side, the other as its negative side. (The points of $p$ are on both sides!) Let $L$ be the set of functions $f$ in $C(E)$ for which there is a point $x$ in the plane with $f(p)=1$ when $x$ is on the positive side of $p$ and $f(p)=-1$ when $x$ is on the negative side of $p$. If $x$ is not on any of the pseudolines, it gives rise to a unique such function $f . L$ is a lopsided set, and this is not difficult to verify, using the definition or Theorem 4.

In fact, this lopsided set comes from an oriented matroid, as in Theorem 7 thanks to the correspondence between arrangements of pseudolines and oriented matroids of rank 3 described by Folkman and Lawrence [4]. (This correspondence extends, in higher dimensions, to one between the "arrangements of pseudo-hemispheres" and the oriented matroids.)

Consider, now, the arrangement $E$ of pseudolines in The Figure, and the corresponding lopsided set $L$. (Grünbaum viewed this arrangement as being in the projective plane. Here it is considered to be in the Euclidean plane, however.) We will see that $L$ is not realizable.

Suppose, on the contrary, that there is a convex set $K \subset V(E)$ with $L=L(K)$. Consider the regions $A, B$, and $C$ of the arrangement, and the associated points $f, g$, and $h$ of $L$.

Suppose $L^{\prime}$ is a lopsided set with:

$$
\{f, g, h\} \subset L^{\prime} \subset L .
$$

We will show that $L^{\prime}=L$.

Consider the smallest face of the cube containing $f$ and $g$; $f$ and $g$ are antipodal on this face. The set $L$ intersects this face in vertices of the cube which form a simple path from $f$ to $g$. By Lemma 1 , all of these vertices must be in $L^{\prime}$, as well. Similarly, $L^{\prime}$ contains the vertices of $L$ which are on the smallest face containing $g$ and $h$, as well as those on the smallest face containing $f$ and $h$. These vertices of $L$ account for all the unbounded regions in the arrangement.

Now, suppose $p$ is the point of intersection of two pseudolines $l_{1}$ and $l_{2}$ of the arrangement. We will see that all four vertices of $L$ corresponding to the four regions incident with $p$ must be in $L^{\prime}$. Let $B=\left\{l_{1}, l_{2}\right\}$, and let $A=E \sim\left\{l_{1}, l_{2}\right\}$. Note that there is a unique function $\alpha$ in $C(A)$ with $\{\alpha\} \times C(B) \subset L$; namely, the function $\alpha=\alpha_{0}$, where:

$$
\alpha_{0}(e)= \begin{cases}1 & \text { if } p \text { is on the positive side of } e \\ -1 & \text { if } p \text { is on the negative side of } e .\end{cases}
$$


There is no function $\beta$ in $C(B)$ with $C(A) \times\{\beta\} \subset C(E) \sim L^{\prime}$, since the points of $L$ corresponding to unbounded regions are in $L^{\prime}$, and for any $\beta \in C(B)$, one of these is in $C(A) \times\{\beta\}$. Since $L^{\prime}$ is lopsided, there must be a function $\alpha$ in $C(A)$ with $\{\alpha\} \times C(B) \subset L^{\prime}$. Clearly, $\alpha=\alpha_{0}$, and the four vertices of $L$ corresponding to the four regions incident with $p$ - the vertices of $\{\alpha\} \times C(B)$ - are all in $L^{\prime}$. Since $p$ was chosen arbitrarily, $L^{\prime}=L$.

Let $r, s$, and $t$ be points of the vector space $V(E)$ with $r \in O(f) \cap K$, $s \in O(g) \cap K$, and $t \in O(h) \cap K$. Let $K^{\prime}$ be the convex hull of $\{r, s, t\}$. Then $\{f, g, h\} \subset L\left(K^{\prime}\right) \subset L$, so $L\left(K^{\prime}\right)=L$.

Consider now the plane containing $K^{\prime}$. It intersects each coordinatehyperplane in a line. These lines form an arrangement of lines $E^{\prime}$ in the plane which yield the lopsided set $L$; but this means $E^{\prime}$ is equivalent to $E$. Since $E$ is not stretchable, this cannot be the case.

It would be interesting to know if there are smaller examples of non-realizable lopsided sets. This was an example of such a set, in a 9-dimensional cube; but Grünbaum proved that the arrangement used is not stretchable in the projective plane, so we may re-draw the arrangement with one of the nine pseudolines "at infinity," and obtain an arrangement of eight pseudolines which is not stretchable (in the Euclidean plane). If we put the line $l$ at infinity, there will again be only three "triangles at infinity," which we may use in place of $A, B$, and $C$, above, to show that the associated lopsided set is not realizable. Are there any non-realizable lopsided subsets of the 7-dimensional cube?

Finally, we mention one last question. Although there are non-realizable lopsided sets, it may still be true that any lopsided set may be derived from an appropriate oriented matroid.

Conjecture. Suppose $L$ is a lopsided subset of the vertex-set of the cube $C$. Then there is a set $L^{\prime}$ contained in the vertex set of a cube $C^{\prime}$, having $C$ as a face, such that:

(1) $L^{\prime}$ is symmetric;

(2) If $F$ is any facet of $C^{\prime}, F \cap L$ is lopsided; and

(3) $L^{\prime} \cap C=L$.

In this case, $L^{\prime}$ comes from a simple oriented matroid, as in Theorems 8 and 9.

ACKNOWLEDGEMENT. We greatly appreciated the encouragement and editorial comments of Alan Goldman during the preparation of this paper. 


\section{REFERENCES}

1. R. G. Bland and M. Las Vergnas, Orientability of matroids, J. Combinatorial Theory, Series B, 24 (1978), 94-123.

2. D. Z. Djoković, Distance-preserving subgraphs of hypercubes, J. Combinatorial Theory, Series B, 14 (1973), 263-267.

3. J. Edmonds and D. R. Fulkerson, Bottleneck Extrema, in Studies in Optimization (ed. Dantzig and Eaves), Studies in Mathematics, Vol. 10, MAA (1974), 94-103.

4. J. Folkman and Jim Lawrence, Oriented matroids, J. Combinatorial Theory, Series B, 25 (1978), 199-236.

5. B. Grünbaum, Arrangements and Spreads, Regional Conf. Series in Math., Number 10, Amer. Math. Soc., (1972).

6. F. Levi, Die Teilung der projektiven Ebene durch Gerade oder Pseudogerade, Ber. math.-phys. Kl. sächs. Akad. Wiss. Leipzig, 78 (1962), 256-267.

7. G. Ringel, Teilungen der Ebene durch Geraden oder Topologische Geraden, Math. Z., 64 (1955), 79-102.

Received September 19, 1981. This work was done while the author was a National Academy of Sciences - National Research Council Postdoctoral Research Associate at the National Bureau of Standards, Washington, D. C. 20234.

UNIVERSITY OF KENTUCKY

LEXINGTON, KY 40506 



\section{PACIFIC JOURNAL OF MATHEMATICS \\ EDITORS}

DONALD BABBITT (Managing Editor)

University of California

Los Angeles, CA 90024

\section{Hugo Rossi}

University of Utah

Salt Lake City, UT 84112

C. C. Moore and Arthur Ogus

University of California

Berkeley, CA 94720
J. DugundiI

Department of Mathematics

University of Southern California

Los Angeles, CA 90089-1113

R. FINN and H. SAMELSON

Stanford University

Stanford, CA 94305

ASSOCIATE EDITORS
R. ARens
E. F. BECKENBACH
B. H. NeumanN
F. WOLF
K. YoshidA (1906-1982)

\section{SUPPORTING INSTITUTIONS}

UNIVERSITY OF ARIZONA

UNIVERSITY OF BRITISH COLUMBIA

CALIFORNIA INSTITUTE OF TECHNOLOGY

UNIVERSITY OF CALIFORNIA

MONTANA STATE UNIVERSITY

UNIVERSITY OF NEVADA, RENO

NEW MEXICO STATE UNIVERSITY

OREGON STATE UNIVERSITY
UNIVERSITY OF OREGON

UNIVERSITY OF SOUTHERN CALIFORNIA

STANFORD UNIVERSITY

UNIVERSITY OF HAWAII

UNIVERSITY OF TOKYO

UNIVERSITY OF UTAH

WASHINGTON STATE UNIVERSITY

UNIVERSITY OF WASHINGTON 


\section{Pacific Journal of Mathematics}

Vol. 104, No. 1

May, 1983

Nestor Edgardo Aguilera and Eleonor Ofelia Harboure de Aguilera, On

the search for weighted norm inequalities for the Fourier transform $\ldots \ldots .1$

Jin Akiyama, Frank Harary and Phillip Arthur Ostrand, A graph and its complement with specified properties. VI. Chromatic and achromatic numbers ......................................... 15

Bing Ren Li, The perturbation theory for linear operators of discrete type . . . 29

Peter Botta, Stephen J. Pierce and William E. Watkins, Linear

transformations that preserve the nilpotent matrices .............. 39

Frederick Ronald Cohen, Ralph Cohen, Nicholas J. Kuhn and Joseph

Alvin Neisendorfer, Bundles over configuration spaces .......... 47

Luther Bush Fuller, Trees and proto-metrizable spaces . . . . . . . . . . 55

Giovanni P. Galdi and Salvatore Rionero, On the best conditions on the

gradient of pressure for uniqueness of viscous flows in the whole space . . 77

John R. Graef, Limit circle type results for sublinear equations $\ldots \ldots \ldots \ldots 85$

Andrzej Granas, Ronald Bernard Guenther and John Walter Lee,

Topological transversality. II. Applications to the Neumann problem for

$y^{\prime \prime}=f\left(t, y, y^{\prime}\right) \ldots \ldots \ldots \ldots \ldots \ldots \ldots \ldots \ldots \ldots \ldots \ldots \ldots \ldots \ldots . \ldots 5$

Richard Howard Hudson and Kenneth S. Williams, Extensions of

theorems of Cunningham-Aigner and Hasse-Evans . . . . . . . . . . . 111

John Francis Kurtzke, Jr., Centralizers of irregular elements in reductive algebraic groups

James F. Lawrence, Lopsided sets and orthant-intersection by convex

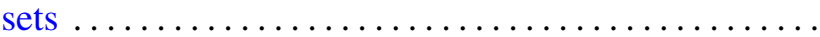

Åsvald Lima, G. H. Olsen and U. Uttersrud, Intersections of $M$-ideals and

$G$-spaces

Wallace Smith Martindale, III and C. Robert Miers, On the iterates of derivations of prime rings

Thomas H. Pate, Jr, A characterization of a Neuberger type iteration procedure that leads to solutions of classical boundary value problems

Carl L. Prather and Ken Shaw, Zeros of successive iterates of multiplier-sequence operators

Billy E. Rhoades, The fine spectra for weighted mean operators

Rudolf J. Taschner, A general version of van der Corput's difference theorem

Johannes A. Van Casteren, Operators similar to unitary or selfadjoint ones 